tung gilt auch für Menschen, die zu einer Risikogruppe gehören können: „Vor allem die Fehlalarme führen zu unnötigen, auch belastenden Abklärungsuntersuchungen." Es werden keine Hinweise auf einen Nutzen, aber solche auf geringe Schäden gesehen.

Eindrucksvoll bestätigt dieser Fall, dass es für den Arzt das Schwierigste ist, eine Handlung zu unterlassen. Mit den Zielsetzungen des IGeL-Katalogs ist das unvereinbar, aber richtig. Der ausufernde Aktionismus, der seine Wurzel in der klinischen Ausbildung an Kranken hat, setzt sich in der Praxis fort, wo er sich im ausgeworfenen IGeL-Netz als Wert- schöpfungskette niederschlägt. Damit schwindet das noch vorhandene Vertrauen der Patienten, die eines guten $\mathrm{Ra}-$ tes, nicht aber einer überflüssigen (Be-) Handlung bedürfen.

Wenn es darum geht, die Gesundheit im Alter zu fördern, dann ist in diesem Fall die Beratung angemessen - die vorgeschlagenen Maßnahmen aus dem IGeL-Katalog sind es nicht. Mein ärztliches Geschäftsmodell sieht einen 3,5-fachen Satz der GOÄ-Nummer 3 vor $30,60 €$ gegenüber 333,00 €. Im Gespräch werde ich das Altern erklären und dafür Akzeptanz schaffen. Damit würde dem 85-Jährigen vieles erspart.
Was bleibt nun aber von einem Beitrag übrig, der geschrieben und akzeptiert wurde, um ausschließlich den betriebswirtschaftlichen Erfolg einer urologischen Praxis als Unternehmensziel zu propagieren? Die Empfehlung, mit dem eigenen moralischen Kompass abzuwägen, zwischen dem was notwendig und dem was möglich ist. Außerdem sollten Früherkennungsleistungen aus dem IGeL-Katalog altersbegrenzt werden. Es wäre gut, an dieser Stelle die internationale Diskussion unter dem Stichwort „preventing overdiagnosis“ fortzusetzen.

Prof. Dr. med. Lothar Weißbach, Gesundheitsforschung für Männer, Berlin

\title{
Leserbrief von Dr. Elke Heßdörfer
}

unge Frauen wie in Fall 2 (38 Jahre,
weiblich, rezidivierende Harnwegsinfektionen) mit der häufigsten urologischen Erkrankung in dieser Altersgruppe, nämlich rezidivierenden Harnwegsinfekten (rHWI), als „auswärtige“ Patientinnen - laut dem Kollegen meint er damit Frauen, die bisher wegen der rHWI bei Gynäkologen oder Hausärzten

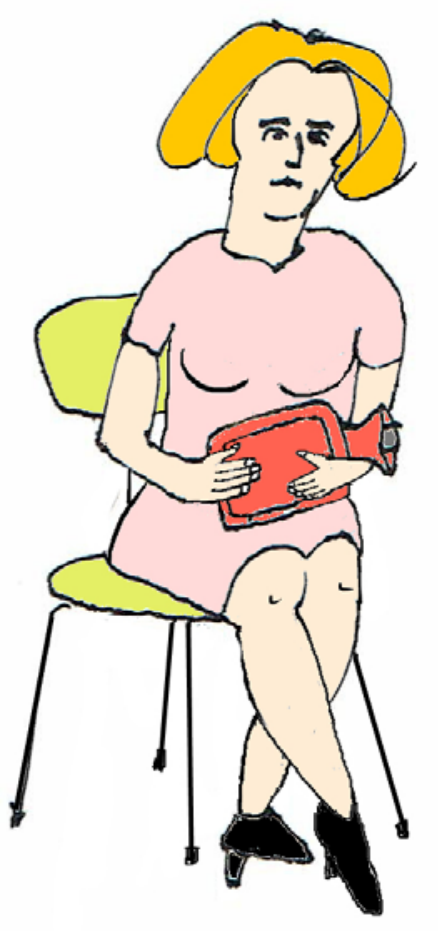

in Behandlung waren - zu deklarieren, nur weil sie kompetenten Rat bei einem Urologen suchen, klingt befremdlich.

Noch bizarrer wird die Argumentation zur Rechtfertigung für das IGeL-Angebot: „Die Anspruchshaltung und der Beratungsbedarf der Patientin mit HWI, die zur Zweitmeinungsberatung kommt, sprengen deutlich den Rahmen der allgemeinen Sprechstunde." Wohlgemerkt: Besagte Patientin war bisher noch nie beim Urologen! Und weiter: „Der Arzt sollte sofort offen kommunizieren, dass die gewünschte Beratung und anschließende Untersuchung inklusive der sinnvollerweise zugehörigen Sonografie des Harntrakts keine Leistungen sind, die durch die gesetzliche Krankenversicherung (GKV) abgedeckt werden. Folgerichtig und berufsrechtlich korrekt ist daher, die erweiterte Diagnostik nach der Gebührenordnung der Ärzte (GOÄ) zu liquidieren." - Genau durch solche Aussagen sind IGeL so in Verruf geraten!

Mein Rat an dieser Stelle: Wenn ein Kollege die RLV-Pauschale im GKV-Bereich zu unrentabel hält - was sie ja zweifelsohne auch ist -, dann wäre er gut beraten seine Kassenzulassung aufzugeben. Andernfalls wäre es gegenüber der ratsuchenden Frau geradezu unethisch, ihr eine Beratung und Sonografie der Nieren und Harnwege als IGeL-Leistung anzubieten. Die Blasen- und durchaus auch die Restharnsonografie sind mit Sicher- heit keine IGel-Leistungen. Das wäre so, also ob man bei einer Nierenkolik die Nierensonografie als IGeL-Leistung anbietet. Ich verweise hierzu auf die aktuelle EAU-Guidelineempfehlung zu rHWI: "An extensive routine workup including cystoscopy, imaging, etc. is not routinely recommended as the diagnostic yield is low. However, it should be performed without delay in atypical cases, for example if renal calculi or outflow obstruction is suspected." Letzteres ist aber bei fehlendem Restharn, sofern man diese Untersuchung zuvor getan hat, bei der ansonsten gesunden Frau nicht anzunehmen. [...] Noch deutlicher wird in der AWMFLeitlinie formuliert: „Bei einer retrospektiven Studie von Frauen mit rezidivierenden Harnwegsinfektionen unterstützten die Daten den routinemäßigen Einsatz einer Ultraschalluntersuchung von Nieren und Restharn“. Daher empfiehlt die Leitliniengruppe: „Bei Patientinnen mit rezidivierenden Harnwegsinfektionen sollte eine Urinkultur und einmalig eine Sonografie erfolgen“" (B, LoE 1b).

Die Therapieempfehlung erscheint mir recht dürftig, da offensichtlich nur eine Immunisierung mit StroVac ${ }^{\circledast}$, das in der jüngsten Studie genauso gut wie Placebo abschnitt, angeboten wird. Hierfür eine Beratungsziffer 1 nach GOÄ inklusive Gebührenordnungspositionen 5 und 375 (allerdings wenn, dann schon bitte dreimal!) zu berechnen, ist völlig legitim, da keine GKV-Leistung. [...]

Dr. med. Elke Heßdörfer, Blasenzentrum Westend, Berlin 